\title{
BMJ Open Intermittent preventive treatment of pregnant women in Kintampo area of Ghana with sulphadoxine- pyrimethamine (SP): trends spanning 2011 and 2015
}

Felix Boakye Oppong, ${ }^{\oplus 1}$ Stephaney Gyaase, ${ }^{1}$ Charles Zandoh, ${ }^{1}$ Obed Ernest A Nettey, ${ }^{1}$ Seeba Amenga-Etego, ${ }^{1}$ Edward Apraku Anane, ${ }^{1}$ Robert Adda, ${ }^{1}$ David Kwame Dosoo, ${ }^{1}$ Seth Owusu-Agyei, ${ }^{1,2}$ Kwaku Poku Asante ${ }^{1}$

To cite: Oppong FB, Gyaase S, Zandoh C, et al. Intermittent preventive treatment of pregnant women in Kintampo area of Ghana with sulphadoxine-pyrimethamine (SP): trends spanning 2011 and 2015. BMJ Open 2019;9:e027946. doi:10.1136/ bmjopen-2018-027946

- Prepublication history for this paper is available online. To view these files, please visit the journal online (http://dx.doi. org/10.1136/bmjopen-2018027946).

Received 26 November 2018 Revised 18 March 2019 Accepted 24 May 2019

Q Check for updates

(c) Author(s) (or their employer(s)) 2019. Re-use permitted under CC BY-NC. No commercial re-use. See rights and permissions. Published by BMJ.

${ }^{1}$ Kintampo Health Research Centre, Kintampo, Ghana ${ }^{2}$ Institute of Health Research, University of Health and Allied Sciences, Ho, Ghana

\section{Correspondence to} Dr Kwaku Poku Asante; kwakupoku.asante@kintampohrc.org

\section{ABSTRACT}

Objective In Ghana, intermittent preventive treatment during pregnancy with sulphadoxine-pyrimethamine (IPTp-SP) is recommended for the prevention of malaria-related adverse outcomes. This study demonstrates the coverage of IPTp-SP use among pregnant women over a period (2011-2015) and the impact of various sociodemographic groups on the uptake of IPTp-SP.

Design Retrospective analysis using data from all pregnant women in the Kintampo Health and Demographic Surveillance System area on the uptake of IPTp-SP.

Setting Kintampo North Municipality and Kintampo South District of Ghana.

Participants All pregnant women in the Kintampo Health and Demographic Surveillance System area.

Primary and secondary outcome measures The number of doses of IPTp-SP taken by pregnant women were examined. Logistic regression was used to assess the determinant of uptake of IPTp-SP while adjusting for within-subject correlation from women with multiple pregnancies.

Results Data from 2011 to 2015 with a total of 17484 pregnant women were used. The coverage of the recommended three or more doses of IPTp-SP among all pregnant women was $40.6 \%, 44.0 \%, 45.9 \%, 20.9 \%$ and $32.4 \%$ in 2011, 2012, 2013, 2014 and 2015, respectively. In the adjusted analysis, age, household size, education, religion, number of antenatal care visits, ethnicity, marital status, wealth index and place of residence were significantly associated with the uptake of three or more doses of IPTp-SP. Having middle school education or higher, aged 20 years and above, visiting antenatal care five times or more (OR 2.83, $95 \% \mathrm{Cl} 2.64$ to 3.03 ), being married (OR $1.10,95 \% \mathrm{Cl} 1.02$ to 1.19) and those in higher wealth quintiles were significantly more likely to take three or more doses of IPTp-SP.

Conclusion The uptake of the recommended three or more doses of IPTp-SP is low in the study area. We recommend a community-based approach to identify women during early pregnancy and to administer IPTp-SP.

\section{INTRODUCTION}

Sub-Saharan Africa is the region that carries the heaviest malaria burden in the world. ${ }^{1}$
Strengths and limitations of this study

- This study used data from the Kintampo Health and Demographic Surveillance System by including all identified pregnant women in the study area over a 5-year period to assess how coverage of intermittent preventive treatment during pregnancy with sulphadoxine-pyrimethamine (IPTp-SP) has changed over the past few years.

- Also, because the entire population of pregnant women in the study area were included, there is no bias in terms of selection of respondents.

- The study findings can be generalised to the Kintampo Health and Demographic Surveillance System area and not the whole of Ghana.

- Although no pregnant woman refused participation, it is possible that a handful of pregnant women will be missed due to the approach used in identifying pregnant women.

- The study was limited to finding the maternal characteristics associated with IPTp-SP.

Among pregnant women in the region, malaria is often the cause of mild to severe anaemia and placental parasitaemia. ${ }^{2}$ Malaria in pregnancy is also associated with low birth weight, as well as increase in the risk of perinatal mortality. ${ }^{2}$ Likewise, malaria infection during pregnancy is an important cause of spontaneous abortion and preterm delivery in the region. ${ }^{1}$ Due to the decreased immunity during pregnancy, pregnant women are usually more vulnerable to malaria infection; it has been reported that they are four times more likely to get the disease and have a doubled chance of dying as a result of malaria. ${ }^{3}$ In Ghana, about $28 \%$ of all outpatient department (OPD) attendance by pregnant women is due to malaria; about $13.7 \%$ of hospital admissions among pregnant women 
is a result of malaria, while $9 \%$ of maternal deaths is attributed to malaria. ${ }^{1}$

Prevention and effective management of malaria during pregnancy helps in reducing the risk of poor outcomes for both mother and baby. For this reason, in the year 2000, the WHO recommended that pregnant women in malaria-endemic countries be given intermittent preventive treatment (IPT) by taking sulphadoxine-pyrimethamine (SP) at least twice at scheduled antenatal visits from 16 weeks of gestation or at quickening. ${ }^{1}$ Use of IPTp-SP among pregnant women has contributed to about $42 \%$ reduction in low birth weight, $38 \%$ reduction in neonatal death and $65 \%$ reduction in placental malaria. ${ }^{4}$ However, the benefits of IPTp-SP have been found to be more pronounced when pregnant women take more doses. Taking three or more doses of IPTp-SP during pregnancy has been found to result in higher mean birth weight, fewer low birth weight as well as less placental malaria, compared with taking at most two doses. ${ }^{5}$ Based on these evidence, revisions were made by WHO in 2012 with recommendation of increasing the frequency of administering IPTp-SP to pregnant women at each antenatal care (ANC) visit until delivery. ${ }^{5}$ In 2013, the Ghana National Malaria Control Programme (NMCP) revised its policy, recommending at least three doses of IPTp-SP ${ }^{6}$ and have since 2014 been implementing it. The Ghana NMCP's strategic plan for 2005-2015 had targeted that, by the year 2015, all pregnant women should receive two or more doses of IPTp-SP, which is now administered from 16 weeks of gestation onwards or at quickening and follow-up doses given on monthly basis.

Over the years, the coverage of at least three doses of IPTp -SP in Ghana has been on the increase, from $28 \%$ in 2008 to $60 \%$ in $2016 .{ }^{6}$ Regardless of the relatively high coverage in the uptake of three or more doses of SP by pregnant women in the country, the gap between uptake of only one dose of IPTp-SP and multiple doses remains significant. For instance, in 2016, the coverage of one dose of IPTp-SP was $85 \%$ compared with the $60 \%$ coverage of at least three doses of IPTp-SP. ${ }^{6}$

This manuscript reports the coverage of IPTp-SP among pregnant women and factors that are associated with the uptake of at least one dose and three or more doses of IPTp-SP using data collected between 2011 and 2015 as part of the Kintampo Health and Demographic Surveillance System (KHDSS). ${ }^{7}$ The findings are expected to serve as evidence to guide the NMCP in the design of appropriate policies and strategies that can help increase the use of IPTp-SP and eventually reduce malaria-related adverse outcomes during pregnancy.

\section{METHOD}

\section{Study area and data description}

Data from the KHDSS were used. The KHDSS, which is managed by the Kintampo Health Research Centre, routinely collects health data from residents of Kintampo North Municipality and Kintampo South District of
Ghana. These are two contiguous districts located in the Brong Ahafo Region and lie in the geographical centre of the country. ${ }^{7}$ With a total population of about 154341 , it covers an area of 7162 square kilometres. ${ }^{8}$ There are 2 hospitals, 5 private clinics, 13 health centres and 43 community-based health planning and services that provide ANC and other healthcare need of the people. KHDSS routinely collects data on several events including pregnancies, births, deaths and migrations, as well as demographic information such as household socioeconomic status, education and causes of death among others. $^{7}$

As part of the routine visits by the KHDSS, in 2011, 2012 and 2013, all registered households were visited three times in a year in a cycle that gave rise to a visit in every 4 months. In 2014 and 2015, six monthly updated visits were made to all registered households. At each visit, household heads (or in the absence, an adult member of the household who was present) were asked by trained field workers if any member of their household was pregnant. Subsequently, identified pregnant women were interviewed by our trained field workers on a one-on-one basis without interference from other household members. Pregnancy registration forms that contained closed-ended questions were used to collect data on basic characteristics of respondents, pregnancy history and outcomes, use of antimalarial during pregnancy, insecticide-treated net (ITN) use and ANC attendance records. Likewise, after birth, study participants were visited, and a birth registration form was used to document birth information and to also collect complete records of the pregnancy data. On the use of IPTp-SP, study participants were asked whether they took antimalarial (IPTp-SP) at any ANC visit under the supervision of a healthcare attendant. Records on the number of times they received IPTp-SP and the corresponding dates of medication were transcribed from their ANC cards. All participants who were 18 years and above individually consented. For participants who were below 18 years, parental consent and assent were obtained. Participation was voluntary since individuals had the right to refuse to be part of the study before or during the interview.

Data on the use of IPTp-SP during pregnancy from 2011 to 2015 were our main focus with the main outcome variable being the number of doses of IPTp-SP taken by each pregnant woman and was dichotomised as: (1) no dose versus at least one dose and (2) less than three doses versus three or more doses. For the predictor variables, maternal characteristics, namely, age, education, use of ITNs, religion, ethnicity, first or subsequent pregnancy, number of ANC visits, household size, place of residence (rural or urban), marital status and wealth index were used.

\section{Patients and public involvement}

The study did not recruit patients. Neither pregnant women nor the public were involved in the development of the research question or design of this study. Key 
stakeholder meeting will be organised to share the findings with the community and Ghana Health Service who are in charge of healthcare delivery in the country.

\section{Statistical analysis}

Cleaned data were analysed using STATA V.14.0. An overall wealth index was computed for each pregnant woman using principal component analysis as presented in Asante et $a l^{9}{ }^{9}$ The computation of the wealth index was based on the number and type of assets (such as televisions, cars, electricity, toilet facilities and house ownership) available in a pregnant woman's household. Pregnant women were grouped by their wealth indices into wealth quintiles namely, poorest, more poor, poor, less poor and least poor. Sociodemographic characteristics of the study participants were summarised using frequencies and percentages.

A univariate logistic regression model was considered for each of the predictor variables to study if they are independently associated with IPTp-SP use. Based on results of past studies, ${ }^{10-15}$ and interest in all the selected sociodemographic characteristics, we included all the predictor variables in a multiple logistic regression model regardless of their statistical significance in the univariate analysis. Two different analyses were performed. First, factors associated with the use of at least one dose of IPTp-SP were determined. This was followed by assessing the factors that were associated with the uptake of three or more doses of IPTp-SP. Considering the period under study, $21.3 \%$ of the study participants had more than one pregnancy. Hence, the data used for the analysis had repeated observations for participants who had more than one pregnancy between 2011 and 2015. In accounting for the fact that data from the same subject are not independent, generalised estimating equation ${ }^{16}$ was used to obtain population-averaged estimates and to address the correlation present in the data. Robust SEs were obtained to alleviate the bias associated with possible misspecification of the correlation structure. With less than $8 \%$ missing data, complete case analysis was conducted by including subjects for whom all the variables involved in the analysis were observed.

\section{RESULTS}

\section{Characteristics of the study population}

A total of 17484 pregnant women were identified from 2011 to 2015 . This was made up of 4065 pregnant women in 2011, 4570 in 2012, 4547 in 2013, 4295 in 2014 and 3870 in 2015. All the identified pregnant women willingly participated in the study. Over the 5-year period, $78.68 \%$ (13 756/17 484) of the pregnant women contacted had one pregnancy; the remaining $21.32 \%$ (3728/17 484) who had multiple pregnancies were made up of $20.55 \%(3593 / 17484)$ with two pregnancies and $0.77 \%(135 / 17484)$ with three pregnancies. Details of the characteristics of the study participants are presented in table 1 .
Factors associated with uptake of at least one dose of IPTpSP

The coverage of at least one dose of IPTp-SP over the 5 -year period is presented in figure 1 . There were significantly higher coverage of at least one dose of IPTp-SP in 2012, 2013 and 2015, compared with 2011, but lower coverage in 2014 (table 2).

Table 2 presents the results of the association between all the maternal characteristics under study and the uptake of at least one dose of IPTp-SP. Age of mother, household size, education, religion, being pregnant for the first time, number of ANC visits, ethnicity, marital status and place of residence were independently associated with the use of IPTp-SP. However, in the adjusted analysis, factors that were significantly associated with the uptake of at least one dose of IPTp-SP were religion of participant, order of pregnancy, number of ANC visits, ethnicity and place of residence (table 2).

\section{Factors associated with uptake of three or more doses of IPTp-SP}

The coverage of the recommended three or more doses of IPTp-SP was $40.6 \%, 44.0 \%, 45.9 \%, 20.9 \%$ and $32.4 \%$ in 2011, 2012, 2013, 2014 and 2015, respectively (figure 1). Compared with 2011, the coverage of three or more doses of IPTp-SP was significantly lower in 2014 and 2015. However, for 2012 and 2013, the coverage of three or more doses of IPTp-SP was significantly higher compared with the coverage in 2011 (table 3).

All the maternal characteristics under study were independently associated with the uptake of the recommended three or more doses of IPTp-SP (table 3). Howver, after adjusting for all other factors, being pregnant for the first time and use of ITNs were not significantly associated with the uptake of three or more doses of IPTp-SP. The detailed results of the factors associated with the uptake of three or more doses of IPTp-SP is presented in table 3.

\section{DISCUSSION}

The coverage of IPTp-SP, as well as the maternal characteristics associated with the use of at least one dose of IPTp-SP and three or more doses of IPTp-SP was assessed among pregnant women in Kintampo North Municipality and Kintampo South District of Ghana. The target set by Ghana National Malaria Control Program was for all pregnant women to be on IPTp-SP by the year $2015 .{ }^{17}$ Although the coverage of at least one dose of IPTp-SP has remained high, the target set by the Ghana National Malaria Control Program was not achieved in Kintampo North Municipality and Kintampo South District.

Compared with the coverage of at least one dose of IPTp-SP, the coverage of the recommended three or more doses of IPTp-SP was lower. Also, there was no significant increase in the uptake of the recommended three or more doses of IPTp-SP after the implementation of the new policy, which required pregnant women to take at least three doses of IPTp-SP. ${ }^{6}$ Rather, the coverage of at 
Table 1 Characteristics of study subjects by year of interaction

\begin{tabular}{|c|c|c|c|c|c|}
\hline \multirow[b]{2}{*}{ Variable } & \multicolumn{5}{|l|}{ Year } \\
\hline & $2011(n=4065)$ & $2012(n=4570)$ & $2013(n=4547)$ & $2014(n=4295)$ & $2015(n=3870)$ \\
\hline \multicolumn{6}{|l|}{ Age of mother (years) } \\
\hline $12-19$ & $12.18(495)$ & $12.21(558)$ & $12.93(588)$ & $13.36(574)$ & 13.00 \\
\hline 20-29 & $45.98(1869)$ & $46.74(2136)$ & $49.44(2248)$ & $47.10(2023)$ & $44.13(1708)$ \\
\hline $30-39$ & 34.49 (1402) & 34.49 (1576) & $32.31(1469)$ & $33.20(1426)$ & 35.45 (1372) \\
\hline$\geq 40$ & $7.36(299)$ & $6.56(300)$ & $5.32(242)$ & $6.33(272)$ & $7.42(287)$ \\
\hline \multicolumn{6}{|l|}{${ }^{*}$ Household size } \\
\hline One member & $14.46(588)$ & $17.51(800)$ & $19.51(887)$ & $18.39(790)$ & $18.60(720)$ \\
\hline Small & $29.25(1189)$ & $28.71(1312)$ & $28.83(1311)$ & $30.31(1302)$ & $29.66(1148)$ \\
\hline Medium & $40.54(1648)$ & $38.99(1782)$ & $37.39(1700)$ & $37.58(1614)$ & $37.13(1437)$ \\
\hline Large & $15.74(640)$ & $14.79(676)$ & $14.27(649)$ & $13.71(589)$ & $14.60(565)$ \\
\hline \multicolumn{6}{|l|}{ †Education } \\
\hline No formal education & $53.11(2159)$ & $50.61(2313)$ & $47.57(2163)$ & 44.75 (1922) & $43.02(1665)$ \\
\hline Primary & $23.30(947)$ & 24.38 (1114) & $25.62(1165)$ & $25.96(1115)$ & 26.05 (1008) \\
\hline Middle/JHS & $19.43(790)$ & $20.94(957)$ & $21.35(971)$ & $22.93(985)$ & $24.52(949)$ \\
\hline Secondary+ & $4.16(169)$ & $4.07(186)$ & $5.45(248)$ & $6.36(273)$ & $6.41(248)$ \\
\hline \multicolumn{6}{|l|}{ Religion } \\
\hline Christian & $55.60(2260)$ & $54.20(2477)$ & $54.52(2479)$ & $53.81(2311)$ & 56.98 (2205) \\
\hline Muslims & 29.69 (1207) & $29.43(1345)$ & 28.79 (1309) & $31.04(1333)$ & $29.25(1132)$ \\
\hline Traditional & $2.48(101)$ & 2.76 (126) & $2.49(113)$ & $2.84(122)$ & $2.53(98)$ \\
\hline No religion & $6.67(271)$ & $6.59(301)$ & $6.55(298)$ & $6.82(293)$ & $6.69(259)$ \\
\hline Missing data & $5.56(226)$ & $7.02(321)$ & 7.65 (348) & $5.49(236)$ & $4.55(176)$ \\
\hline First pregnancy & 25.39 (1032) & $24.90(1138)$ & $25.12(1142)$ & $25.26(1085)$ & $22.25(861)$ \\
\hline Use of ITNs & $54.51(2216)$ & 65.69 (3002) & 77.68 (3532) & $80.23(3446)$ & 77.83 (3012) \\
\hline \multicolumn{6}{|l|}{ Number of ANC visits } \\
\hline 0 or 1 visit & 6.99 (284) & 6.39 (292) & $7.15(325)$ & $6.43(276)$ & $7.05(273)$ \\
\hline $2-4$ visits & $44.94(1827)$ & $44.73(2044)$ & $44.64(2030)$ & $44.77(1923)$ & 42.95 (1662) \\
\hline 5 or more visits & 48.07 (1954) & $48.88(2234)$ & $48.21(2192)$ & $48.80(2096)$ & 50.00 (1935) \\
\hline \multicolumn{6}{|l|}{ †Ethnicity } \\
\hline Akan & $19.58(796)$ & $20.63(943)$ & $20.23(920)$ & $20.84(895)$ & $21.14(818)$ \\
\hline Northern Tribes & $57.96(2356)$ & $57.40(2623)$ & $57.69(2623)$ & $57.88(2486)$ & $57.78(2236)$ \\
\hline Mo & $12.05(490)$ & $10.96(501)$ & $11.55(525)$ & $11.94(513)$ & $12.07(467)$ \\
\hline Others & 7.77 (316) & $7.59(347)$ & $6.82(310)$ & $7.22(310)$ & $6.87(266)$ \\
\hline Missing data & $2.63(107)$ & 3.41 (156) & $3.72(169)$ & $2.12(91)$ & $2.14(83)$ \\
\hline \multicolumn{6}{|l|}{ Marital status } \\
\hline Married & 48.51 (1972) & $47.35(2164)$ & $45.22(2056)$ & $43.66(1875)$ & $45.40(1757)$ \\
\hline Not married & $51.49(2093)$ & $52.65(2406)$ & $54.78(2491)$ & $56.34(2420)$ & $54.60(2113)$ \\
\hline \multicolumn{6}{|l|}{ Wealth index } \\
\hline Poorest & $25.58(1040)$ & $21.49(982)$ & $20.91(951)$ & $15.37(660)$ & $20.70(801)$ \\
\hline More poor & $23.30(947)$ & $21.18(968)$ & $21.33(970)$ & $19.23(826)$ & $21.42(829)$ \\
\hline Poor & $22.16(901)$ & $20.57(940)$ & $20.67(940)$ & 19.09(820) & $19.77(765)$ \\
\hline Less poor & $17.93(729)$ & $19.50(891)$ & $19.68(895)$ & $19.72(847)$ & $19.92(771)$ \\
\hline Least poor & $10.80(439)$ & $16.98(776)$ & $17.29(786)$ & $26.33(1131)$ & $17.83(690)$ \\
\hline Missing data & $0.22(9)$ & $0.28(13)$ & $0.11(5)$ & $0.26(11)$ & $0.36(14)$ \\
\hline
\end{tabular}


Table 1 Continued

\begin{tabular}{llllll}
\hline \multirow{2}{*}{ Variable } & \multicolumn{1}{l}{ Year } & & & \\
\cline { 2 - 6 } & $\mathbf{2 0 1 1}(\mathbf{n}=\mathbf{4 0 6 5 )}$ & $\mathbf{2 0 1 2}(\mathbf{n}=\mathbf{4 5 7 0})$ & $\mathbf{2 0 1 3} \mathbf{( n = 4 5 4 7 )}$ & $\mathbf{2 0 1 4}(\mathbf{n}=\mathbf{4 2 9 5})$ & $\mathbf{2 0 1 5}(\mathbf{n}=\mathbf{3 8 7 0})$ \\
\hline Rural & $68.29(2776)$ & $64.81(2962)$ & $65.60(2983)$ & $66.78(2868)$ & $66.72(2582)$ \\
Urban & $31.71(1289)$ & $35.19(1608)$ & $34.40(1564)$ & $33.22(1427)$ & $33.28(1288)$ \\
\hline
\end{tabular}

Data are presented as percentage (frequency).

${ }^{*}$ Small (2-4 people), medium (5-10 people) and large (more than 10 people).

†Secondary+ (secondary school education or higher) and junior high school (JHS).

$\ddagger$ Northern tribes (tribes from any of the three Northern regions of Ghana) and other (any other ethnic group).

ANC, antenatal care; JHS, junior high school; ITNs, insecticide-treated net.

least one dose as well as three or more doses of IPTp-SP in 2014 and 2015 was lower compared with the period preceding the implementation of the new policy. The low coverage of the recommended three or more doses of IPTp-SP has also been reported in other parts of the country. ${ }^{18-20}$ Challenges associated with the implementation of the new policy may have contributed to the low IPTp-SP coverage in 2014 and 2015. In 2014, transportation challenges as well as delays in allocating malaria commodities affected the distribution of SP to most periphery facilities in Ghana. This resulted in artificial stock outs of SP in several facilities within the country. ${ }^{212}$ To help improve coverage, Ghana NMCP should ensure that at all times, all heath facilities in the country have SP in stock. Also, the low uptake of the recommended three or more doses of IPTp-SP compared with the national coverage could be due to contextual factor that might have influenced the implementation of the new policy. There is the need to further explore these contextual factors. However, this finding emphasises the variations in IPTp uptake in Ghana and the need for context-specific interventions especially in rural settings such as the study area.

Several maternal sociodemographic characteristics were found to be associated with the uptake of at least

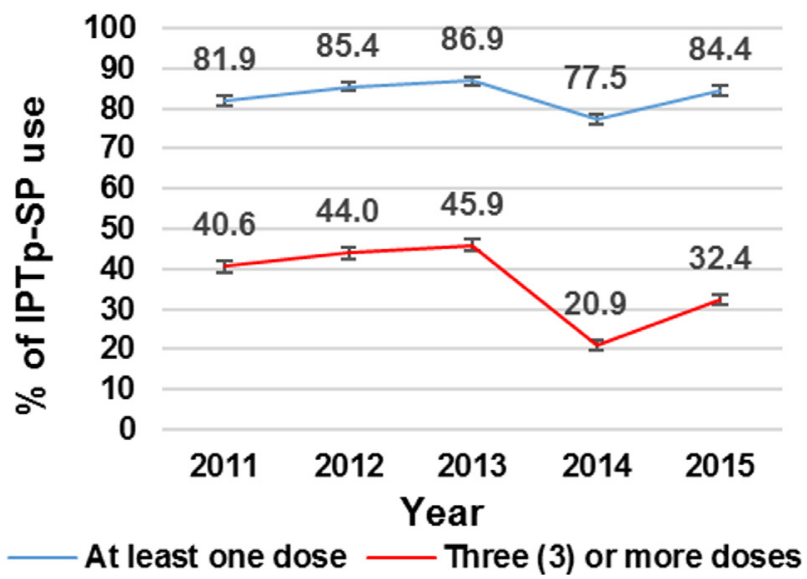

Figure 1 Doses of IPTp-SP taken by pregnant women in the Kintampo North Municipality and Kintampo South District from 2011 to 2015. IPTp-SP, intermittent preventive treatment during pregnancy with sulphadoxinepyrimethamine. one dose of IPTp-SP. With regards to order of pregnancy, as expected, women who have subsequent pregnancies were more likely to take at least one dose of IPTp-SP. This may be due to their knowledge and education on the benefits of IPTp-SP gained from previous pregnancies. Undoubtedly, frequency of ANC attendance was found to be significantly associated with the uptake of at least one dose of IPTp-SP. This is not surprising given that IPTp-SP is a directly observed therapy administered during ANC visits.

The low uptake of the recommended three or more doses of IPTp-SP among teenage participants compared with older participants can be attributed to the low patronage of ANC among teenagers. Due to the stigma associated with teenage pregnancy in Ghana, pregnant teenagers are less likely to go for ANC compared with older pregnant women. ${ }^{23} 24$ Often, teenagers hide their pregnancies and delay in ANC attendance ${ }^{24}$ and are therefore not able to take the recommended doses before they deliver. Given the increased risks faced by pregnant adolescents, we recommend that ANC clinics in the study area should be made adolescent friendly and should also integrate health education on the benefits of ANC visits and uptake of IPTp-SP. Likewise, parents and guardians should assist pregnant adolescents and encourage them to attend ANC as well as to educate them on the burden of malaria infection during pregnancy and the need to take the recommended number IPTp-SP doses.

Similar to the results on the uptake of at least one dose of IPTp-SP, the number of times a woman attends ANC was significantly associated with the uptake of the recommended three or more doses of IPTp-SP. A pregnant woman can only take the recommended three or more doses of IPTp-SP if she attends ANC at least three times. In Ghana, it is recommended for all pregnant women to attend ANC at least four times. ${ }^{523}$ Even so, the new policy on the uptake of IPTp-SP recommends that starting as early as the beginning of the second trimester of pregnancy, SP should be administered at each scheduled ANC visit until delivery. Likewise, the policy stipulates that except in the first trimester, IPTp-SP can be administered every month with subsequent doses given at least 1 month apart. This implies that more doses of IPTp-SP are recommended. Given these recommendations, it is important 
Table 2 Factors associated with use of at least one dose of IPTp-SP

\begin{tabular}{lllll}
\hline Predictor & Unadjusted OR $\mathbf{9 5 \%} \mathbf{C l})$ & P value & Adjusted OR $(\mathbf{9 5} \% \mathbf{C l})$ & P value \\
\hline Age of mother (years) & & & & \\
$12-19$ & 1 & & 1 & \\
$20-29$ & $1.12(1.01$ to 1.25$)$ & 0.044 & $1.00(0.87$ to 1.15$)$ & 0.999 \\
$30-39$ & $1.22(1.09$ to 1.37$)$ & 0.001 & $1.06(0.90$ to 1.25$)$ & 0.485 \\
$\geq 40$ & $1.11(0.93$ to 1.31$)$ & 0.242 & $1.01(0.81$ to 1.24$)$ & 0.959
\end{tabular}

Household size

One member

1

Small 1

Medium

0.95 (0.85 to 1.06$)$

0.350

1.00 (0.88 to 1.12$) \quad 0.939$

Large

0.87 ( 0.78 to 0.97$)$

0.010

0.98 ( 0.87 to 1.11$)$

0.795

Education

None

0.90 (0.79 to 1.03$)$

0.120

1.03 (0.89 to 1.19$)$

0.698

Primary

Middle/JHS

Secondary+

Religion

Christian

Muslims

Traditional

No religion

First pregnancy

No

Yes

Use of ITNs

No

Yes

1.02 (0.94 to 1.10$)$

1

0.89 (0.82 to 0.96$)$

1.02 (0.81 to 1.29$)$

0.88 (0.76 to 1.02 )

1

0.86 (0.79 to 0.93 )

$<0.001$

0.220

0.001

0.874

1.08 (0.95 to 1.22$)$

0.803

0.85 (0.69 to 1.03 )

0.227

0.101

1

0.86 (0.78 to 0.95$) \quad 0.003$

1.20 (0.93 to 1.53$) \quad 0.158$

0.98 (0.83 to 1.15$)$

0.771

0.091

1

$0.84(0.75$ to 0.94$) \quad 0.002$

Number of ANC visits

0 or 1 visit

2-4 visits

8.71 (7.71 to 9.82$)$

$<0.001$

8.87 (7.80 to 10.08$)$

$<0.001$

5 or more visits

5.48 (4.88 to 6.15$)$

$<0.001$

5.22 (4.60 to 5.92$)$

$<0.001$

Ethnicity

Akan

Northern Tribes

1

Mo

0.93 (0.85 to 1.02$)$

1.18 (1.03 to 1.35$)$

Others

0.92 (0.79 to 1.07 )

Marital status

Not married

Married

1

Wealth index

Poorest

More poor

Poor

1.05 (0.94 to 1.17$)$

Less poor

Least poor

Place of residence
1

1.05 (0.93 to 1.18$) \quad 0.475$

1.19 (1.03 to 1.39$) \quad 0.021$

0.93 (0.78 to 1.12$) \quad 0.456$

$0.295 \quad 0.93(0.78$ to 1.12$) \quad 0.456$

1

$0.012 \quad 1.01(0.92$ to 1.11$) \quad 0.842$ 
Table 2 Continued

\begin{tabular}{|c|c|c|c|c|}
\hline Predictor & Unadjusted OR $(95 \% \mathrm{Cl})$ & $P$ value & Adjusted OR (95\% Cl) & $P$ value \\
\hline Rural & 1 & & 1 & \\
\hline Urban & 1.50 (1.39 to 1.63$)$ & $<0.001$ & $1.56(1.42$ to 1.72$)$ & $<0.001$ \\
\hline 2011 & 1 & & 1 & \\
\hline 2012 & 1.28 (1.14 to 1.43$)$ & $<0.001$ & $1.24(1.10$ to 1.41$)$ & 0.001 \\
\hline 2014 & 0.77 (0.69 to 0.86$)$ & $<0.001$ & $0.72(0.64$ to 0.81$)$ & $<0.001$ \\
\hline 2015 & 1.21 (1.08 to 1.36$)$ & 0.001 & 1.19 (1.05 to 1.36$)$ & 0.008 \\
\hline
\end{tabular}

IPTp-SP, intermittent preventive treatment during pregnancy with sulphadoxine-pyrimethamine.

ANC, antenatal care; JHS, junior high school; ITNs, insecticide-treated nets.

for pregnant women to initiate ANC visit as early as possible in order to be able take the required doses of SP.

Strategies such as Transforming IPT for Optimal Pregnancy (TIPTOP) initiative can be adopted in the study area to help increase IPTp-SP coverage. The approach of TIPTOP involves engaging community health volunteers to identify women during early stages of pregnancy, to educate them on the benefits of early ANC attendance and IPTp-SP use and to refer them to a health facility for ANC. Also, with the goal of increasing IPTp-SP coverage, volunteers of the TIPTOP initiative distribute SP to pregnant women at the community level. ${ }^{25}$ This approach to administering IPTp-SP will help increase coverage by reaching out to all pregnant women irrespective of their sociodemographic characteristic. In a study conducted in Uganda, community-based delivery of IPTp-SP was found to increase IPTp-SP coverage. Also, the administration of IPTp-SP at the community level resulted in a reduction in the prevalence of malaria parasitaemia, severe anaemia and low birth weight and was acceptable to about $90 \%$ of women who intend to use SP in the future. ${ }^{26}$ In addition to this approach, studies have reported that provision of incentives to pregnant women during ANC visits is associated with an increase in ANC attendance. ${ }^{27}{ }^{28}$ Hence, we propose the provision of some form of non-monetary incentives to pregnant women who attend all scheduled ANC visits to help motivate other pregnant women to attend all scheduled ANC which may eventually lead to an increase in the coverage of IPTp-SP.

The low uptake of the recommended three or more doses of IPTp-SP among unmarried participants compared with married participants could be attributed to the possible support that married women get from their partners during pregnancy. In most rural settings in Ghana such as our study area, women largely depend on their husbands for financial support in seeking healthcare. ${ }^{29}$ Married women may have received the necessary financial support to travel to attend ANC for the recommended three or more doses of IPTp-SP considering that frequency of ANC attendance was associated with uptake of the recommended doses of IPTp-SP. The high uptake of the recommended three or more doses of IPTp-SP among educated participants compared with those with no formal education can also be attributed to how informed women with formal education are about the increased risk of malaria infection during pregnancy and the complications associated with malaria during pregnancy. ${ }^{30}$ This further reiterates the significant role education plays with regards to the use of health interventions. ${ }^{30}$ Other studies in Ghana ${ }^{10}$ and other parts of Africa including Uganda ${ }^{12}$ and Kenya ${ }^{13} 14$ also reported an association between education and the uptake IPTp-SP.

One would expect that due to the knowledge gained from past pregnancies on the importance of malaria prevention during pregnancy and the benefits of IPTp$\mathrm{SP}$, women with history of past pregnancies will take the recommended three or more doses of IPTp-SP. However, even though participants who were pregnant for the first time were less likely to take at least one dose of IPTp-SP, there was no significant association between history of past pregnancy and uptake of the recommended three or doses of IPTp-SP. Other studies in Ghana ${ }^{31}$ and other parts of Africa including Nigeria ${ }^{32}$ and Cameroon ${ }^{33}$ have also reported no association between subsequent pregnancies and uptake of the recommended doses of IPTpSP. In Ghana, association has been reported between wealth and health, such that people with better socioeconomic status have better health-seeking behaviour. ${ }^{34}$ This is reiterated in this study given that women in the higher wealth quintiles are more likely to take the recommended three or more doses of IPTp-SP. Even though IPTp-SP is provided to pregnant women free of charge, they are only given to women when they visit a health facility for ANC care. Sometimes, cost/means of transportation to the nearest ANC clinic prevent pregnant women from attending ANC. ${ }^{2435} 36$ As such, women in the higher wealth quintiles are more likely to attend ANC since they may be able to bear the cost of visit to ANC clinics. ${ }^{36}$ This result is similar to that reported in the most recent Ghana Demographic and Health Survey in which the coverage of the recommended three or more doses of IPTp-SP was $50.6 \%$ and $36.6 \%$ among women in the highest wealth 
Table 3 Factors associated with the uptake of three or more doses of IPTp-SP

\section{Predictor}

Age of mother (years)

12-19

20-29

30-39

$\geq 40$

Household size

One member

Small

Medium

Large

Education

None

Primary

Middle/JHS

Secondary+

Religion

Christian

Muslims

Traditional

No religion

First pregnancy

No

Yes

Use of ITNs

No

Yes

Number of ANC visits

0 or 1 visit

2-4 visits

5 or more visits

1

Ethnicity

Akan

Northern Tribes

Mo

Others

Marital status

Not married

Married

Wealth index

Poorest

More poor

Poor

Less poor

Least poor

Place of residence

1
Unadjusted OR $(95 \% \mathrm{Cl}) \quad \mathrm{P}$ value

\section{Adjusted OR $(95 \% \mathrm{Cl}) \quad$ P value}

1.49 (1.35 to 1.64$)$

$<0.001$

1.46 (1.32 to 1.61$)$

$<0.001$

0.063

1.28 (1.13 to 1.45$)$

$<0.001$

1.15 (0.99 to 1.33$)$

1.35 (1.17 to 1.56$)$

$<0.001$

1.27 (1.05 to 1.53$)$

0.013

1

$0.92(0.84$ to 1.00$) \quad 0.054$

1

1.01 (0.91 to 1.12$) \quad 0.817$

0.83 (0.75 to 0.91$) \quad<0.001$

0.70 (0.64 to 0.76$) \quad<0.001$

0.87 (0.77 to 0.99$)$

0.029

1.05 (0.96 to 1.15$) \quad 0.288$

$1.17(1.05$ to 1.29$) \quad 0.003$

$1.31(1.10$ to 1.56$) \quad 0.002$

$1.53(1.42$ to 1.65$) \quad<0.001$

$<0.001$

1

$\begin{array}{lllr}0.74(0.69 \text { to } 0.80) & <0.001 & 0.83(0.76 \text { to } 0.90) & <0.001 \\ 0.59(0.48 \text { to } 0.71) & <0.001 & 0.83(0.67 \text { to } 1.02) & 0.082\end{array}$

0.64 (0.56 to 0.72$)$

0.83 (0.72 to 0.95$)$

0.007

$<0.001$

1

$1.07(0.97$ to 1.18$) \quad 0.170$

1.09 (1.01 to 1.16$)$

0.018

0.170

0.87 (0.81 to 0.92$)$

1

$\begin{array}{lll}<0.001 & 1.07(0.99 \text { to } 1.16) & 0.076\end{array}$

(

1

$2.83(2.64$ to 3.03$) \quad<0.001$

3.04 (2.85 to 3.23$)$

$<0.001$

1

$\begin{array}{lrrr}0.62(0.57 \text { to } 0.67) & <0.001 & 0.87(0.79 \text { to } 0.97) & 0.010 \\ 0.90(0.81 \text { to } 1.01) & 0.062 & 0.97(0.86 \text { to } 1.10) & 0.646 \\ 0.73(0.64 \text { to } 0.83) & <0.001 & 0.77(0.66 \text { to } 0.90) & 0.001\end{array}$

0.97 (0.86 to 1.10$) \quad 0.646$

0.73 (0.64 to 0.83$)$

$0.008 \quad 1.10(1.02$ to 1.19$) \quad 0.015$

1.09 (1.02 to 1.15$)$

1

1

0.98 (0.89 to 1.08$)$

0.667

$1.03(0.93$ to 1.15$) \quad 0.526$

1.22 (1.11 to 1.34$)$

$<0.001$

1.28 (1.15 to 1.43$)$

$<0.001$

$<0.001$

$1.25(1.12$ to 1.40$) \quad<0.001$

1.40 (1.27 to 1.54$)$

1.32 (1.17 to 1.49 )

$<0.001$

Continued 
Table 3 Continued

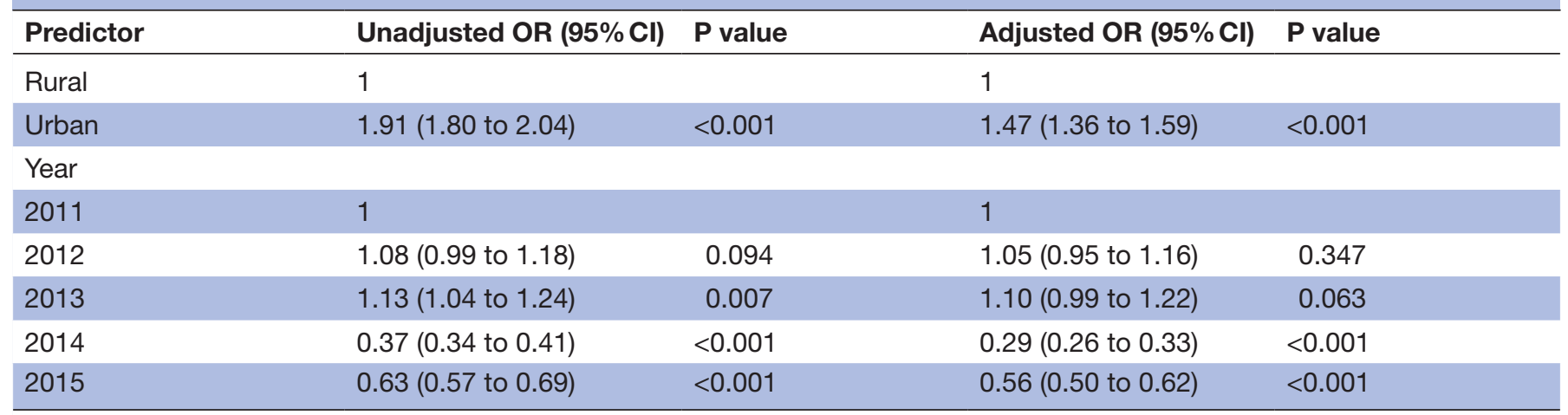

ANC, antenatal care; JHS, junior high school; IPTp-SP, intermittent preventive treatment during pregnancy with sulphadoxinepyrimethamine; ITNs, insecticide-treated nets.

quintile and those in the lowest quintile, respectively. ${ }^{37}$ Also, similar results have been reported in Senegal, ${ }^{38}$ where women in richer or middle wealth quintile were more likely to use the recommended doses of IPTp.

It should be emphasised that the use of data from Health and Demographic Surveillance Systems can be of great importance in informing policy makers of challenges in several health interventions. Particularly, this study used the KHDSS by including all identified pregnant women in the study area over a 5-year period. Unlike the results of cross-sectional studies, the use KHDSS has made it possible to assess and compare the coverage of IPTp-SP over a 5-year period. It is also important to note that, with the use of data from all 156 communities of the KHDSS, the study findings can be generalised to all the KHDSS area and not the whole of Ghana. Also, because this study used the entire population of pregnant women, there is no bias in terms of selection of respondents. Although no pregnant woman refused participation, it is possible that a handful of pregnant women will be missed due to the approach used in identifying pregnant women. Also, the study was limited to finding the maternal characteristics associated with IPTp-SP use.

\section{CONCLUSION}

The use of at least one dose of IPTp-SP in the KHDSS area is generally high. However, uptake of the recommended three or more doses of IPTp-SP remains low. The study has found that the most vulnerable pregnant women are less likely to take the recommended three or more doses of IPTp-SP. The study therefore recommends the adoption of the TIPTOP strategy to help improve the coverage of IPTp-SP in the study area.

Acknowledgements The authors are grateful to the study participants as well as the study community members. We also appreciate the effort of Abukari Mahama, Grace Manu, Francis Agbokey, Francis Dzabeng and Samuel Afari-Asiedu for their participation in the manuscript discussion meetings.

Contributors FBO, KPA and SO-A conceived the idea and conceptualised the study. CZ, OEAN and EAA designed the study instrument and collected the data. SA-E and RA managed the data. The data analysis and interpretation of results were conducted by FBO, SG, DKD and KPA. FBO drafted the manuscript, and SG,
CZ, OEAN, SA-E, EAA, DKD, RA, SO-A and KPA contributed to the revisions of the manuscript. All authors read and approved the final manuscript.

Funding The authors have not declared a specific grant for this research from any funding agency in the public, commercial or not-for-profit sectors.

Competing interests None declared.

Patient consent for publication Not required.

Ethics approval Ethical approval was granted by the Institutional Ethics Committee of the Kintampo Health Research Centre of Ghana.

Provenance and peer review Not commissioned; externally peer reviewed.

Data sharing statement Dataset is not publicly available. Upon request from the corresponding author, details about the statistical code and/or data set can be obtained.

Open access This is an open access article distributed in accordance with the Creative Commons Attribution Non Commercial (CC BY-NC 4.0) license, which permits others to distribute, remix, adapt, build upon this work non-commercially, and license their derivative works on different terms, provided the original work is properly cited, appropriate credit is given, any changes made indicated, and the use is non-commercial. See: http://creativecommons.org/licenses/by-nc/4.0/.

\section{REFERENCES}

1. Ghana Health Service (GHS). Malaria in pregnancy training manual for health providers.

2. Desai M, ter Kuile FO, Nosten F, et al. Epidemiology and burden of malaria in pregnancy. Lancet Infect Dis 2007;7:93-104.

3. Groves J, El-Shirbiny D. Revision Notes for the DRCOG: A Textbook of Women's Health: CRC Press, 2015.

4. Garner P, Gulmezoglu A,et al. Drugs for preventing malaria in pregnant women. Cochrane Database Syst Rev 2006;4.

5. World Health Organization (WHO). WHO policy brief for the implementation of intermittent preventive treatment of malaria in pregnancy using sulfadoxine-pyrimethamine (IPTp-SP. Geneva: World Health Organization, 2013.

6. Ghana Statistical Service (GSS), Ghana Health Service (GHS), and ICF. Ghana Malaria Indicator Survey 2016. Accra, Ghana, and Rockville, Maryland, USA: GSS, GHS, and ICF, 2017.

7. Owusu-Agyei S, Nettey OE, Zandoh C, et al. Demographic patterns and trends in Central Ghana: baseline indicators from the Kintampo Health and Demographic Surveillance System. Glob Health Action 2012;5:19033.

8. Kintampo Health Reseach Centre (KHRC). Kintampo Health Research Centre Annual Report 2017;2016.

9. Asante KP, Owusu-Agyei S, Cairns M, et al. Placental malaria and the risk of malaria in infants in a high malaria transmission area in ghana: a prospective cohort study. J Infect Dis 2013;208:1504-13.

10. Nwaefuna EK, Afoakwah R, Orish VN, et al. Effectiveness of intermittent preventive treatment in pregnancy with sulphadoxinepyrimethamine against submicroscopic falciparum malaria in central region, Ghana. J Parasitol Res 2015;2015:1-6.

11. Hajira I. Factors Influencing Uptake of Intermittent Preventive Treatment of Malaria in Pregnancy using Sulphadoxine 
Pyrimethamine in the Sunyani Municipality. Ghana: University of Ghana, 2015.

12. Kiwuwa MS, Mufubenga P. Use of antenatal care, maternity services, intermittent presumptive treatment and insecticide treated bed nets by pregnant women in Luwero district, Uganda. Malar J 2008;7:44.

13. Eijla A, Ayisi G, Kuile F. Implementation of IPT with SP for control of malaria in Kisumu, Kenya. Malar J 2002:265-6.

14. Ouma PO, Van Eijk AM, Hamel MJ, et al. The effect of health care worker training on the use of intermittent preventive treatment for malaria in pregnancy in rural western Kenya. Trop Med Int Health 2007;12:953-61.

15. Marchant $\mathrm{T}$, Nathan $\mathrm{R}$, Jones $\mathrm{C}$, et al. Individual, facility and policy level influences on national coverage estimates for intermittent preventive treatment of malaria in pregnancy in Tanzania. Malar $J$ 2008;7:260.

16. LIANG K-YEE, Zeger SL. Longitudinal data analysis using generalized linear models. Biometrika 1986;73:13-22.

17. Owusu-Boateng I, Anto F. Intermittent preventive treatment of malaria in pregnancy: a cross-sectional survey to assess uptake of the new sulfadoxine-pyrimethamine five dose policy in Ghana. Malar J 2017;16:323.

18. Doku DT, Zankawah MM, Adu-Gyamfi AB. Factors influencing dropout rate of intermittent preventive treatment of malaria during pregnancy. BMC Res Notes 2016;9:460.

19. Tutu EO, Lawson B, Browne E. The effectiveness and perception of the use of sulphadoxine-pyrimethamine in intermittent preventive treatment of malaria in pregnancy programme in Offinso district of Ashanti region, Ghana. Malar J 2011;10:385.

20. Hommerich L, von Oertzen C, Bedu-Addo G, et al. Decline of placental malaria in southern Ghana after the implementation of intermittent preventive treatment in pregnancy. Malar J 2007;6:144

21. Odjidja EN. Low Uptake of Intermittent Preventive Treatment in Ghana; An Examination of Health System Bottlenecks. Archivos De Medicina 2017;4:60.

22. Ghana National Malaria Control Program (GNMC). 2014 Annual Report National Malaria Control Programme, 2015.

23. Ghana Health Service (GHS). Family Health Division Annual Report 2016, 2017.

24. Pell C, Meñaca $A$, Were $F$, et al. Factors affecting antenatal care attendance: results from qualitative studies in Ghana, Kenya and Malawi. PLoS One 2013;8:e53747.

25. JHPIEGO. Community Intermittent Preventive Treatment for Malaria in Pregnancy Implementation Guide Version 2, 2018.
26. Mbonye AK, Bygbjerg I, Magnussen P. Intermittent preventive treatment of malaria in pregnancy: a community-based delivery system and its effect on parasitemia, anemia and low birth weight in Uganda. Int J Infect Dis 2008;12:22-9.

27. Khogali M, Zachariah R, Reid AJ, et al. Do non-monetary incentives for pregnant women increase antenatal attendance among Ethiopian pastoralists? Public Health Action 2014;4:12-14.

28. Till SR, Everetts D, Haas DM. Incentives for increasing prenatal care use by women in order to improve maternal and neonatal outcomes. Cochrane Database Syst Rev 2015:CD009916.

29. Opoku G. Review of Maternal Mortality and Near-Miss Events in Kintampo Municipality in the Brong Ahafo Region of Ghana. Texila International Journal of Public Health. 2016;4.

30. Hill J, Hoyt J, van Eijk AM, et al. Factors affecting the delivery, access, and use of interventions to prevent malaria in pregnancy in sub-Saharan Africa: a systematic review and meta-analysis. PLoS Med 2013;10:e1001488.

31. Ibrahim H, Maya ET, Issah $\mathrm{K}$, et al. Factors influencing uptake of intermittent preventive treatment of malaria in pregnancy using sulphadoxine pyrimethamine in Sunyani Municipality, Ghana. Pan Afr Med J 2017;28.

32. Amoran OE, Ariba AA, lyaniwura CA. Determinants of intermittent preventive treatment of malaria during pregnancy (IPTp) utilization in a rural town in Western Nigeria. Reprod Health 2012;9:12.

33. Takem EN, Achidi EA, Ndumbe PM. Use of intermittent preventive treatment for malaria by pregnant women in Buea, Cameroon. Acta Trop 2009;112:54-8.

34. Danso-Appiah A, Stolk WA, Bosompem KM, et al. Health seeking behaviour and utilization of health facilities for schistosomiasisrelated symptoms in ghana. PLoS Negl Trop Dis 2010;4:e867.

35. Andrew EV, Pell C, Angwin A, et al. Factors affecting attendance at and timing of formal antenatal care: results from a qualitative study in Madang, Papua New Guinea. PLoS One 2014;9:e93025.

36. Arthur E. Wealth and antenatal care use: implications for maternal health care utilisation in Ghana. Health Econ Rev 2012;2:14.

37. Ghana Statistical Service, Ghana Health Service (GHS), ICF International. Ghana Demographic and Health Survey 2014. Rockville, Maryland, USA: GSS, GHS, and ICF International, 2015. Available at. http://www.statsghana.gov.gh/docfiles/publications/ 2014\%20GDHS\%20\%20Report.pdf. (Accessed on 29 Sep 2018).

38. Mbengue MAS, Bei AK, Mboup A, et al. Factors influencing the use of malaria prevention strategies by women in Senegal: a crosssectional study. Malar J 2017;16:470. 